There is evidence that more than $90 \%$ of the matter in our universe is non-luminous in that it neither emits nor reflects detectable electromagnetic radiation. This highly abundant but unknown form of matter is called dark matter. Revealing itself only via gravitational interactions, it was discovered by astronomers who carefully measured the rotational velocities of stars around the centres of spiral galaxies and the movements of galaxies in clusters. Understanding the physical nature of dark matter has become one of the greatest challenges facing astrophysicists and particle physicists, stimulating a large number of experiments.

\section{Evidence from Galaxies}

Spiral galaxies

The most impressive evidence for dark matter comes from measuring the orbital velocities $V$ of luminous matter around the centres of spiral galaxies using image-intensifier techniques to determine the spectral Doppler shifts along a galactic diameter. The velocities as a function of the radial distance $r$ from the galactic centre were obtained for more than 100 spiral galaxies [Rubin et al., 1978]. The result was surprising: the orbital velocities in all cases remained constant out to the far edges of the galaxies, in apparent contradiction to velocity distributions expected from Kepler's law:

$$
V^{2}(r) \approx M(r) G / r
$$

for a mass inside a sphere with radius $r$ of $M(r)$, and gravitational constant $G$. If luminous (detectable) matter is representative of the total mass of the galaxy, one expects Kepler's law to hold since the luminous matter is mainly concentrated at the centre of the galaxy (Fig. 1). The high orbital velocities at large radial distances provide convincing evidence that the mass of the galaxy is not concentrated at the galactic centre. Indeed, numerical analyses of the orbital velocities point to $M(r)$ increasing with $r$ (i.e., the fraction of dark to luminous matter is increasing with increasing radial distance from the galactic centre).

Observations as well as theoretical arguments suggest that the dark matter is distributed in the form of a spherical halo around the galactic disc. In estimating how far the halo extends one notes that many spiral galaxies are surrounded by hydrogen gas which reaches out far beyond the visible disc. It has been possible to measure the orbital velocity of this gas by the radioastronomical observation of the $21 \mathrm{~cm}$ line [Roberts, 1975; Sancisi and von Albada et

Klaus Pretzl is the Director of the HighEnergy Physics Laboratory at the University of Bern, Sidlerstrasse 5, CH-3012 Bern, where he was appointed as a professor in 1988. He received his doctorate from the University of Munich in 1969 before working at Fermilab in the USA until 1973, when he joined the Max-Planck-Institut für Physik, Munich.

\title{
Bringing Dark Matter in from the Dark
}

\author{
K.P. Pretzl \\ Laboratory for High-Energy Physics, University of Bern, Switzerland
}

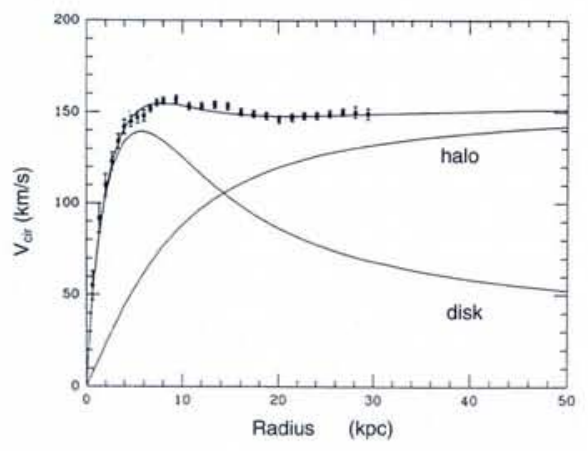

Fig. 1-Orbital velocities as a function of the distance from the centre of the spiral galaxy NGC 3198. The dots with error bars are the observed values and the solid lines are theoretical fits allowing for a halo of dark matter and a luminous disc of maximum mass. [From van Albada et al., 1985].

al., 1985]. Constant orbital velocities out to radial distances corresponding to several times the visible galactic diameter were found, in good agreement with optical measurements (Fig. 2). However, there are no hints indicating how far the dark matter halos extend.

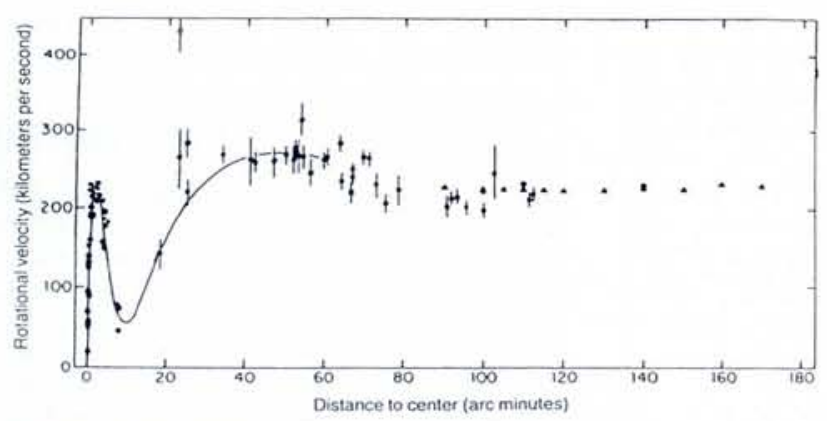

Fig. 2 - The rotational speed as a function of the radial distance for M31, the Andromeda Galaxy, from optical observations by Rubin and Ford (1970) and from radio observations (21 cm line) by Roberts et al.
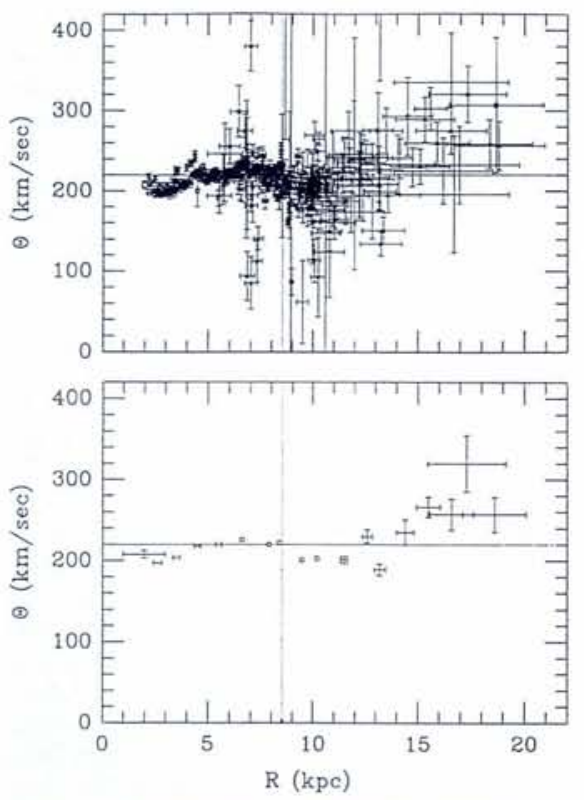

Fig. 3 - The rotational speed of our galaxy as a function of the radial distance: the data (upper) and averaged values (lower). The lines show the position and orbital velocity of the Sun. (From [1]).
The orbital velocities have also been determined [1] in our Milky Way (Fig. 3) in spite of the measurements being much more difficult. The uniform velocity distribution implies that our own galaxy possesses a dark halo. Recently, astronomers from the University of California measured the speed of our nearestneighbour galaxy, the Large Magellanic Cloud, as it spins around us. This speed turns out to be higher than it would be if there exists only the gravitational attraction of the visible mass of our galaxy. The data indicate that the dark halo mass is 6-8 times larger than the visible mass, implying that we can search for dark matter in our own galaxy with Earth-bound detectors.

\section{Elliptical galaxies}

Concerning other types of galaxies, the motion of the stars in elliptical galaxies is irregular and any rotation that is present appears to be small. So the Doppler-shift technique cannot be applied in the same way as for spiral galaxies. However, certain ellipticals are surrounded by a hot plasma gas emitting $x$-rays which can be used to determine a galaxy's gravitational potential. Assuming that the gas is in hydrodynamic equilibrium, the virial theorem relates the kinetic energy of the gas to its gravitational binding energy $(2 T+U=0$ for a kinetic energy $T$ and a gravitational binding energy $U)$. X-ray observations show that the plasma gas has sufficient kinetic energy to escape from the galaxy unless there is much more than just the visible matter which gravitationally binds the gas to the galaxy. However, it should be noted that the validity of the equilibrium argument is still debated. 


\section{Mass estimates}

In order to estimate the amount of dark matter in a galaxy one needs to determine the amount of luminous matter. This is in principle possible by measuring the luminosity and the mass-to-light ratio $Y=M / L$ $\left(M_{\odot} / L_{\odot}\right)$ which is usually given in units of $M_{\odot} / L_{\odot}$, where $M_{\odot}=2 \times 10^{33} \mathrm{~g}$ is the solar mass and $L_{\odot}=3.9 \times 10^{33} \mathrm{erg} \mathrm{s}^{-1}$ is the solar luminosity. The mass-to-light ratio of a stellar population depends sensitively on the number density of the particular types of stars in the population. If a system of stars is primarily composed of massive main sequence stars then $Y<1$, whereas if stars of low mass or low luminosity dominate the population then $Y>1$. So the mass of a stellar population can only be estimated from its luminosity by making certain assumptions. In addition, the luminosity of distant galaxies depends on the value of the Hubble constant $H_{0}$ relating the redshifts seen in the spectra of galaxies outside our own cluster (the Local Group) to these galaxies' distances from us. While $H_{0}\left(=100 h_{0} \mathrm{~km} \mathrm{~s}^{-1}\right.$ $\mathrm{Mpc}^{-1}$ with $0.4<h_{0}<1.0$ ) is not known to better than a factor of 2 , the ratio $Y$ can fortunately be calibrated for some stellar systems where the mass is known from independent measurements.

Fitting the rotation curves of spiral galaxies to a total gravitating mass consisting of a dark matter halo and a luminous disc gives typical values of $Y_{\mathrm{V}} \approx 30 h_{0}$ for the visible spectral band. However, if the dark halo is not taken into account then $Y_{V} \approx 1.5$ for the luminous matter in the galactic disc near our solar system. The results therefore indicate that spiral galaxies have about 10 times more dark than luminous matter.

\section{Galactic Clusters}

\section{Dynamic properties}

Galaxies are not distributed uniformly but clumped into groups comprising a few galaxies, into clusters of many thousands, and even into superclusters. Very convincing evidence for dark matter comes from investigating the dynamic properties of galaxies in large clusters. F. Zwicky pointed out in 1933 that a large amount of dark matter is needed to explain the balance between the gravitational attraction and the motion of galaxies in the Coma cluster. Assuming that the system of galaxies was in hydrodynamic equilibrium, he applied the virial theorem to derive from the measured average velocity of individual galaxies, the total gravitating mass $M$ of the cluster using the relation

$$
M \approx\left\langle V^{2}>R / G\right.
$$

where $R$ is the radius of the cluster and $\langle V\rangle$ is the mean velocity. $\mathrm{He}$ found values of $M$ for the Coma cluster which were 400 times larger than the sum of the masses of the galaxies in the cluster. These very original, but rather crude, measurements were repeated recently using more refined techniques to yield mass-to-light ratios $Y_{V} \approx$ $360 h_{0}$ for the visible band, and $Y_{\mathrm{B}} \approx 400 h_{\mathrm{O}}$ for the blue band; values of $Y_{V} \approx 300 h_{0}$ were typically obtained for other clusters.

However, two difficulties affect the estimate of the mean velocities. First, there is the question of membership of the cluster. For example, the inclusion of fast-moving galaxies which do not belong to the cluster would increase the estimated $M$. Second, Doppler-shift measurements allow the determination of only one component of the galaxy motion, namely the motion towards or away from an observer. One has therefore to assume that the galaxies in a cluster move at the same speed in all directions (the assumption is probably safe for spherical clusters which are believed to be in hydrodynamic equilibrium). There is also the problem of the effective radius $R$ of the cluster, and here one assumes that dark matter in the cluster is distributed in the same way as the visible matter. If, for example, the dark matter is concentrated in the centre then the total mass $M$ would be underestimated. Nonetheless, all these uncertainties cannot account for the large amount of dark matter contained in the clusters.

The study of galactic clusters has until recently been difficult owing to the long observation times needed to measure the redshifts of many galaxies. Modern fibreoptic techniques now allow the limited observation time allocated to these experiments on otherwise heavily booked telescopes to be used more efficiently. If the positions of the galaxies are well known, the light from each galaxy can be individually guided through an optical fibre to a spectro-

graph so measurements on many galaxies can be made simultaneously.

Another way to determine dark matter in galactic clusters exploits gravitational lensing (see insert) whereby a clump of dark matter in the foreground acts as a gravitational lens, distorting the image of more distant background objects (the deflection of light is proportional to the mass of the lens). Modern charge-coupled devices (CCD's) for detecting light mean that we can observe very distant and faint galaxies, thus enabling searches for clumps of dark matter over a large region of the universe.

Recently, the ROSAT satellite has produced images of clusters and small groups of galaxies using the $x$-rays stemming from the Bremsstrahlung emission of fast moving electrons in the hot intergalactic plasma of clusters. Determinations of the gravitating mass of the clusters from the $\mathrm{x}$-ray data confirm the presence of large amounts of dark matter in the clusters. Secondly, the total mass of intergalactic gas represents less than $20 \%$ of the cluster mass.

\section{Alternative Explanation}

Milgrom and Bekenstein [2] have considered alternative explanations for the constant rotational dependence observed for spiral galaxies. They introduced a new gra-

\section{Gravitational Microlensing}

B. Paczynski proposed in 1986 the idea of using gravitational micro-lensing to detect massive dark halo objects in our own galaxy by monitoring the brightness of individual stars in the Large Magellanic Cloud. The method is based on the fact that the light from a distant star is deflected by the gravitational field of a massive object which is close to the line-of-sight between the star and the observer. This deflection produces multiple images of the star (see figure). However, if the light deflection is very small and below the resolving power of the observing telescope, the star will appear brighter than in the absence of a deflector. For a terrestrial observer watching a distant star, the brightness of the star changes if a dark-matter star passes near the line-of-sight. Detection of baryonic dark matter in the form of brown dwarfs or planetary-sized objects with masses in the range $10^{-6} M_{\odot}$ to $10^{-1} M_{\circ}$ should be possible.

Telescopes with charged-coupled devices as cameras are used to monitor many millions of stars simultaneously for several months. The number of micro-lensing events depends on the number of observed stars the observation time, and the mass of the dark-halo objects. Typical event numbers and the estimated mean micro-lensing times for different deflector masses for an observed sample of $10^{6}$ stars in 4 months observation time are given in the table. The time period over which the brightness of a star changes depends on the mass of the deflector and its velocity normal to the line-of-sight. The calculations assume a simple isothermal model for the mass distribution in the dark halo of our galaxy, and an average viria velocity $\langle V\rangle \approx 270 \mathrm{~km} \mathrm{~s}^{-1}$; the brightness magnification factor was taken to be $>1.34$, which is a very reasonable value for current experiments.

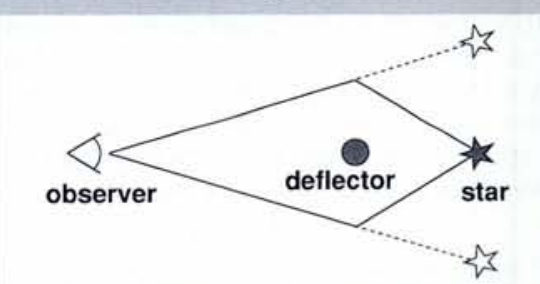

The principle of gravitational lensing. The light of a star is deflected by a point-like deflector and an observer sees two images of the star.

A typical lensing event has the following features: it occurs only once and is not repeated, the light stays acromatic, and the increase and the decrease of the brightness takes place in equal time periods. These signatures allow lensing events to be distinguished from intrinsically variable stars.

Three groups are presently searching for dark matter using micro-lensing (a French group using the European Southem Observatory, a Californian-Australian collaboration using the Mt. Stromlo Observatory, and an American-Polish collaboration using the Los Campañas Observatory in Chile). Recent simultaneous announcements by the first two of candiate events (see insert, page 169) have aroused considerable interest as longterm observations should show either that there is a large number of dark stars or that dark matter is better accounted for in terms of new fundamental particles.

\begin{tabular}{ccc}
\hline \multicolumn{3}{c}{ Micro-lensing events as a function of the } \\
deflector mass
\end{tabular}


vitational acceleration with a constant value of $a_{0}=10^{-8} \mathrm{~cm} \mathrm{~s}^{-2}$. The gravitational acceleration of a test mass by a body of mass $M$ at a distance $r$ is assumed to be

$$
g=\left\{\begin{array}{lll}
g_{N} & \text { if } & g_{N} \gg a_{0} \\
\left(g_{N} a_{0}\right)^{1 / 2} & \text { if } & g_{N} \ll a_{0}
\end{array}\right.
$$

where $g_{N}=G M / r^{2}$ is the gravitational acceleration according to Newton's law. Although this non-relativistic phenomenological approach can explain the observations quite successfully, most cosmologists do not consider it satisfactory, mainly because of the absence of a metric satisfying general relativity.

\section{Density Distribution}

The cosmological significance of dark matter can be seen through the density parameter $\Omega$ defined by the ratio of the mean mass density $\langle\rho\rangle$ to the critical density of the universe $\rho_{c}$, where $\rho_{c}=3 H_{0}^{2} / 8 \pi G$ $=2.8 \times 10^{11} h_{0}{ }^{2} M_{0} M_{p c}^{-3}=1.9 \times 10^{-29} h_{0}{ }^{2}$ $\mathrm{g} \mathrm{cm}^{-3}$. The parameter $\Omega$ determines the fate and geometry of the universe in that $\Omega>1$ results in a closed universe which will eventually collapse, $\Omega=1$ yields a flat universe, and with $\Omega<1$ it expands forever.

The mean mass density of the universe can be estimated from the mass-to-light ratio $Y$ using the relation $\langle\rho\rangle=\langle L\rangle Y$, where $<L>$ is the mean luminosity density which is experimentally determined to be $2.4 \times 10^{8}$ $h_{0} L$. Mpc ${ }^{-3}$ with an uncertainty of a factor of 2. The density parameter is therefore $\langle Y\rangle / Y_{\mathrm{C}}$ where $Y_{\mathrm{c}} \approx 1200 h_{\mathrm{o}}$. Considering only the luminous matter, and using the mass-to-light ratio obtained from the luminous regions of spiral and elliptical galaxies, gives a $\Omega$ value that is slightly less than 0.01 (i.e., the luminous matter in the universe contributes less than $1 \%$ of the critical density). In contrast, the density parameters derived from the flat rotation curves of spiral galaxies are in the range $0.03-0.10$, while the virial velocity measurements of galaxies in clusters correspond to $\Omega$ values of 0.1 0.3 . The data therefore suggest that the universe is open and continually expanding.

\section{A flat universe}

There are, however, theoretical arguments supporting a flat universe. The very high degree of homogeneity and isotropy observed in the cosmic microwave radiation, and the fact that magnetic monopoles are not observed, can be explained by an inflationary scenario for the early universe (originally proposed by A. Guth). This model assumes that $10^{-35} \mathrm{~s}$ after the Big Bang the universe underwent a phase of very rapid expansion which resulted in a flat geometry with $\Omega$ close to 1 . If we believe in inflation, which is presently the case for many cosmologists, one has to expect about 100-times more dark matter than luminous matter in the universe.

Among the attempts to infer $\Omega$ using very large-scale structures by observing the gravitational acceleration of our Local Group owing to large mass concentrations, the survey carried out by the Infra-Red Astronomy Satellite (IRAS) provides the best available information about mass concentrations over a large volume of the universe. The estimated $\Omega$ values range from 0.2 to $\approx 1$. However, the density parameters obtained from IRAS have to be viewed carefully since they depend strongly on assumptions such as how the total mass is distributed with respect to the luminous mass.

\section{Nucleosynthesis}

In order to estimate the amount of baryonic dark matter (the relevent baryons in this case are protons and nuclei) one has to quantify the baryonic density of the universe. Measurements of the cosmic abundances of light elements such as $\mathrm{D},{ }^{3} \mathrm{He}$, ${ }^{4} \mathrm{He}$, and ${ }^{7} \mathrm{Li}$ [3] and the Big Bang Nucleosynthesis (BBN) model constrain the fraction of the critical density due to baryons to $0.01 \leq \Omega_{\mathrm{B}} h_{\mathrm{O}}{ }^{2} \leq 0.02$, although it should be noted that $\Omega_{\mathrm{B}}$ depends on the value of the Hubble constant, which is not accurately known. Even with $h_{0}$ as low as $\approx 0.4$, the baryon fraction works out to be smaller than 0.13 . Alternatives to $B B N$ suggest large inhomogeneities which could have been produced in a first-order quark-hadron transition. It was hoped that such inhomogeneities would allow $\Omega_{\mathrm{B}}$ to be much larger, and perhaps even close to one. However, if agreement with the light-element abundances is sought then inhomogeneous nucleosynthesis adds very little, if anything, to the value of $\Omega_{\mathrm{B}}$ obtained from the BBN model.

A comparison of the baryonic density with the matter density in spiral galaxies suggests that a large fraction, if not all, of the dark matter can be of baryonic nature (brown dwarfs, neutron stars or Jupitersized clumps of matter). Matter densities observed in galactic clusters leave enough room for non-baryonic dark matter, even after considering the upper bound of the baryonic density $\Omega_{\mathrm{B}} \leq 0.13$. The strongest arguments for non-baryonic dark matter,

\section{FIRST MACHO CANDIDATES ANNOUNCED}

The French collaboration based at Saclay using micro-lensing to detect dark matter reported at conferences this summer that it has examined $40 \%$ of a 7 million star sample, while the US-Australian collaboration, based at the University of California, Berkeley, has analysed $60 \%$ of 2.8 million sample. If the dark matter halo around the galactic disc is disposed in massive, compact objects (some like to call these MACHOS - Massive Astrophysical Compact Halo Objects) then both collaborations would expect to see three micro-lensing events: the French collaboration have repor- however, comes from the inflationary model of the universe which inspires speculation on new physics beyond the Standard Model of particle physics.

\section{Dark Matter Candidates}

\section{Baryonic dark matter}

If neutron stars account for a significant fraction of the dark matter in galaxies, then almost all the mass of the galaxies would have been produced in supernovae. This in turn implies an abundance of heavy elements, which is incompatible with current observations. Neutron stars are therefore unlikely candidates. Black holes cannot be excluded. However, they require that early in the formation of galaxies a large number of stars was produced with an appropriate mass range to allow the formation of black holes from stellar objects.

Among the other candidates for objects made of baryonic matter there are brown dwarfs, namely stars which are too small to ignite hydrogen burning and are difficult to detect because of their very low luminosities. As their name implies, brown dwarfs are not very massive $\left(<0.08 \mathrm{M}_{\odot}\right)$ and their surface temperature gives them a reddishbrown colour. One hopes to detect these faint objects with infrared telescopes in future satellite missions to learn more about their abundance. It is also conceivable that a large amount of dark matter exists in the form of clumped objects of planetary size (Jupiter). The micro-lensing experiments presently underway (see insert) are searching for these objects in our own galaxy.

\section{Non-baryonic dark matter}

The fact that baryons alone cannot explain dark matter suggests that dark matter exists as weakly interacting massive particles (WIMP's). Non-baryonic dark matter candidates include, for example, neutrinos with non-vanishing mass, axions and particles predicted by theories such as supersymmetry (SUSY) aiming to unify all the forces in Nature.

\section{Neutrinos}

Among the particle candidates the neutrino is the only one known to exist. It comes in three flavours (electron, muon and tau neutrinos). The existence of additional light neutrino flavours has recently been excluded following measurements of the decay width of the intermediate vector boson Z at $\mathrm{e}^{+} \mathrm{e}^{-}$colliders. Many experiments have searched for neutrino masses but so far only upper limits have been determined. The neutrino mass problem will continue to be studied in neutrino oscillation experiments at CERN (CHORUS and NOMAD), and in the new generation of solar neutrino experiments (GALLEX, SAGE, SUPERKAMIOKANDE, BOREXINO, ICARUS, and SNO) because neutrino oscillations provide a possible explanation for the missing neutrinos from the Sun. A necessary condition for neutrino oscillations to occur is the existence of neutrino mass eigenstates.

It is assumed in most Big Bang models that the relic abundance of neutrinos is comparable to that of photons. Thus, if neutrinos have a mass $m_{v}$ they contribute a fraction

$$
\Omega h^{2}{ }_{0} \approx m_{v}\left(n_{v} / n_{\gamma}\right) / 25
$$


to the critical density of the universe, where $n_{v}$ and $n_{\gamma}$ are the number densities of neutrinos and photons, respectively, and $m_{v}$ is in $\mathrm{eV} c^{-2}$. Since $n_{\mathrm{v}} \approx n_{\gamma}$, neutrinos with a mass of $25 \mathrm{eV} \mathrm{C}^{-2}$ would close the universe. In an isothermal dark-halo model, the velocity of dark matter particles in the rest frame of our galaxy is assumed to be given by a Maxwell-Boltzmann distribution with an average value of $\langle V\rangle \approx 270 \mathrm{~km} \mathrm{~s}^{-1}$ and an upper cut-off value of $575 \mathrm{~km} \mathrm{~s}^{-1}$ corresponding to the escape velocity. Thus, if light neutrinos account for the dark matter in our galaxy, they would have a characteristic momentum $p \approx 10^{-2} \mathrm{eV} c^{-1}$ and a kinetic energy $E \approx 10^{-5} \mathrm{eV}$. Unfortunately, a realistic experimental method able to detect neutrinos with such small energies does not exist. However, if a neutrino mass of the order of $25 \mathrm{eV}$ $c^{-2}$ can be established in future experiments the dark matter problem will be solved even without being able to directly detect darkhalo neutrinos.

\section{Axions}

The introduction of axions was not motivated by cosmological considerations. Invented by particle physicists [R. Peccei and $H$. Quinn] to solve the charge conjugationparity problem in strong interactions, they are potent dark matter candidates owing to their abundance. The relic density of nonrelativistic axions is expected to be

$$
\Omega h^{2}{ }_{\mathrm{o}} \approx\left(m_{\mathrm{a}} / 10^{-5}\right)^{-1 / 2}
$$

where $m_{\mathrm{a}}$ is the mass of the axion in eV $c^{-2}$, so axions with a mass of about $10^{-5} \mathrm{eV} \mathrm{c}^{-2}$ would close the universe and they would be very abundant (number density $\approx 10^{9} \mathrm{~cm}^{-3}$ ). Axion searches using an ingenious microwave technique (see insert) have been carried out in the USA.

\section{SUSY particles}

Heavy, stable supersymmetric particles are also attractive candidates. Supersymmetry is a theory of elementary particles in which all fermions have bosonic partners, and all bosons have fermionic ones. For example, the photon's partner is the spin $1 / 2$ photino, the neutrino's partner is the spin 0 sneutrino, and the partners of the quarks are the spin 0 squarks. SUSY particles would have been produced during the early phase transition of the universe, and the lightest - most stable - ones (e.g., the spin $1 / 2$ neutralino - a superposition of photino, zino and higgsino - the photino and the sneutrino) are ideal candidates for dark matter. Their interactions with ordinary matter would have roughly the strength of the weak interaction. Accelerator experiments at CERN and at Fermilab in the USA have searched for SUSY particles without success, suggesting that if these particles exist at all, they are very massive (> $45 \mathrm{GeV}$ $c^{-2}$ ). The search will continue at CERN's planned Large Hadron Collider and at the USA's Superconducting Super Collider.

\section{WIMP's}

The search for WIMP's with laboratory detectors is encouraged by the large expected particle flux. Assuming that all of the dark matter in our galaxy consists of WIMP's one can estimate their flux from the

\section{Detecting Axions}

Axions interact with two photons with a coupling strength proportional to the mass of the axion. Although the natural two-photon decay rate would be much too slow $\left(10^{40}\right.$ years) to be experimentally detectable, by supplying one of the photons from a coherent source such as a magnetic field a significant conversion rate to one photon can be achieved (see figure). Since the virtual photon from the magnetic field has practically no energy, the outgoing real photon carries an energy equal to the sum of the axion rest mass and its kinetic energy. For axion masses in the interesting range of around $10^{-5} \mathrm{eV} \mathrm{c}^{-2}$ the conversion signal is expected to be several $\mathrm{GHz}$. Thus, galactic axions can in principle be detected by placing a microwave cavity in a strong magnetic field and measuring its power output [P. Sikivie, 1983]. However, since the axion mass is unknown one has to scan over a large range of cavity frequencies, with a long integration time at each frequency: this sets practical limitations.

The axion interaction with photons is described by the Lagrangian $L_{\text {ary }}=g_{\text {arr }}$ a $\vec{E} \cdot \vec{B}$ where $g_{\text {ary }}$ is the axion coupling to the two photons, $a$ is the axion field, and $\vec{E}$ and $\vec{B}$ are the electric and magnetic fields, respec-

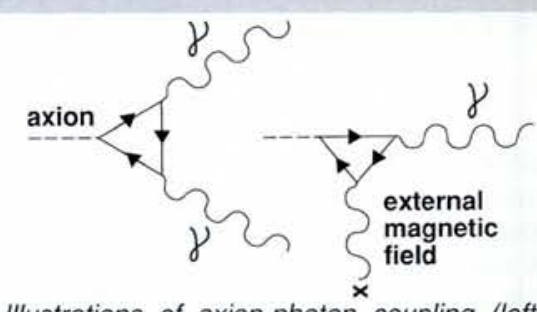

Illustrations of axion-photon coupling (left) and axion-photon conversion in an external magnetic field (right).

tively. The limits of the axion coupling as a function of the axion mass $m_{\mathrm{a}}$ obtained from two axion search experiments are shown in the figure: they hold assuming that the dark halo of our galaxy is made of axions with a local density of $\approx 0.4 \mathrm{GeV} \mathrm{cm}^{-3}$ estimated from the rotation curves. The dashed and solid lines in the figure corresponding to the predictions of two different axion models illustrate that the sensitivity of the experiments was $2-3$ orders of magnitude too low. However, a new experiment plans to use a larger cavity volume and stronger magnetic fields available in the two "axicell" magnets from a decommissioned fusion facility to reach sensitivities below the model predictions.
Upper limits on the axion-two photon coupling as a function of the axion mass $m_{a}$, assuming that the galactic halo is made of axions, for cavity detectors (UF - University of Florida: $R B F$ - Rochester, Brookhaven, Fermilab collaboration). Dashed and solid lines give the sensitivities required to detect galactic halo axions for two different axion models.

local dark matter halo density $\rho \approx 0.4 \mathrm{GeV}$ $\mathrm{cm}^{-3}$ and their average virial velocity $\langle v\rangle$ $\approx 270 \mathrm{~m} \mathrm{~s}^{-1}$ to be

$$
\phi=\rho<v>/ m_{x}=1.08 \times 10^{7} / m_{x}
$$

where $\phi$ is the flux in particles $\mathrm{cm}^{-2} \mathrm{~s}^{-1}$ and $m_{\mathrm{x}}$ is the WIMP mass in GeV $c^{-2}$. The coherent elastic scattering cross-section (neutral current exchange) for a weakly interacting WIMP with a nucleus of the detector material is given approximately by $G_{F}^{2} N^{2} M_{\text {red }}^{2} / 2 \pi$ where $G_{F}$ is the Fermi coupling constant, $N$ is the number of neutrons in the nucleus, and $M_{\text {red }}$ is the reduced mass of the WIMP-nucleus system. The factor $N^{2}$ can be as large as $10^{4}$ for heavy nuclei, and mainly because of this factor the cross-sections become relatively large, leading to appreciable interaction rates with detectors (see insert). The photino, however, being its own antiparticle does not coherently scatter. Its interactions are spindependent (axial vector coupling) and the rates come out to be more like a few events $\mathrm{kg}^{-1}$ day $^{-1}$. Neutralinos have spin-dependent and spin-independent interactions: the rates depend strongly on the assumed supersymmetry parameters so predictions vary by several orders of magnitude. Experiments with sensitivities of about 5 events $\mathrm{kg}^{-1}$ month $^{-1}$ will cover a parameter space which is perhaps large enough to be able to detect neutralinos, if they exist. Nevertheless, as far as dark matter detection is concerned, it is more appropriate to take an exploratory approach rather than paying too much attention to theoretical model predictions.

There is an interesting effect, which will turn out to be important for distinguishing a dark matter signal from background, owing to the movement of the Earth with respect to the dark halo rest frame [4]. Since the orbital velocity of the Earth around the Sun is 30 $\mathrm{km} \mathrm{s}^{-1}$ and the velocity of the Sun around the galactic center is $232 \mathrm{~km} \mathrm{~s}^{-1}$, the Earth will have its maximum velocity of $247 \mathrm{~km} \mathrm{~s}^{-1}$ on June 2 nd and its minimum of $217 \mathrm{~km} \mathrm{~s}^{-1}$ on December 4th, taking the $60^{\circ}$ tilt angle of the ecliptic plane of the Earth with respect to the galactic plane into account. So there is a seasonal modulation of the dark matter detector rates (as large as $10 \%$ depending on the WIMP mass, the detector material, and the detector threshold [5]). Owing to elastic scattering on the constituent nuclei of the Earth, a diurnal modulation of the flux and velocity distribution of WIMP's is predicted [6]. Observable modulations are expected to be a maximum for southern latitudes.

The radioactive background of the detector system itself (cietector material and 


\section{Detecting WIMPS}

As their name implies, WIMP's are weakly interacting particles so the only way to detect them is by measuring the nuclear recoil energy, which is

$$
\Delta E=v^{2}\left(m_{\mathrm{n}} m_{\mathrm{x}}\right)^{2}(1-\cos \theta) /\left(m_{\mathrm{n}}+m_{\mathrm{x}}\right)^{2}
$$

where $m_{\mathrm{n}}$ is the mass of the nucleus, $m_{\mathrm{x}}$ the mass of the WIMP, $v$ the velocity of the WIMP, and $\theta$ the scattering angle in the center-of-mass system. The expected recoil energies for some detectors are shown in the table, where nuclear form factors have been taken into account in calculating interaction rates.

Searches for WIMP's, and also for solar neutrinos, have inspired the development of cryogenic particle detectors to reach much lower energy thresholds and higher energy resolutions than conventional systems. How ever, first experimental limits for WIMP's (see figure) were obtained using $\mathrm{Si}$ and $\mathrm{Ge}$ dete- citors originally designed for double-beta decay experiments, and from $\mathrm{Nal}$ detectors [Bacci C. et al., Phys. Lett. B 239 (1992) $460]$.

Conventional detectors have limited capabilities when recoil energies are small. At low energies, most of the recoil energy is transformed into heat, causing a temperature change $\Delta T=\Delta E / C$ in the detector, where $C$ is the heat capacity of the detector. At low temperatures, where $C$ is very small, even a very small recoil energy $\Delta E$ can be transformed into a measurable signal using superconducting calorimetric devices with low energy thresholds (see insert below), for example. The quantum energy in a superconductor is typically $<1 \mathrm{meV}$ (the energy it takes to break a Cooper pair) and is much smaller than the quantum energies of $20 \mathrm{eV}$ and $1 \mathrm{eV}$ involved in ionization and semiconductor detector devices, respectively.

\section{WIMP interaction rates $\left(\mathrm{kg}^{-1}\right.$ day $\left.^{-1}\right)$ and expected average nuclear recoil energies $\mathrm{keV}$ )}

for various detectors.

\begin{tabular}{|c|c|c|c|c|c|c|}
\hline WIMP mass & & ${ }^{118} \mathrm{Sn}$ & & ${ }^{74} \mathrm{Ge}$ & & ${ }^{28} \mathrm{Si}$ \\
\hline $\mathrm{GeV} c^{-2}$ & Rate & Recoil energy & Rate & Recoil energy & Rate & Recoil energy \\
\hline 1 & 207 & $5 \times 10^{-3}$ & 124 & $8 \times 10^{-3}$ & 34 & $2 \times 10^{-2}$ \\
\hline $\begin{array}{l}100 \\
1000\end{array}$ & $\begin{array}{l}1424 \\
188\end{array}$ & $\begin{array}{l}7.5 \\
12\end{array}$ & $\begin{array}{l}911 \\
125\end{array}$ & $\begin{array}{l}9.8 \\
17\end{array}$ & $\begin{array}{l}133 \\
19\end{array}$ & $\begin{array}{l}8.9 \\
13\end{array}$ \\
\hline
\end{tabular}

surrounding materials) is a common problem for all dark matter detectors. Besides cryogenic detectors, there are plans to use more conventional devices such as ultrapure Nal crystals The Rutherford Appleton Laboratory, UK, is presently involved in preparing a large underground dark matter experiment using both $\mathrm{Nal}$ crystals and cryogenic systems. There are also indirect searches involving the detection of annihilation products of dark matter particles, the most interesting being those trapped in the Sun or Earth. If they are high-energy neutrinos they could be detected, for instance, using underground (MACRO or ICARUS at Gran Sasso) and underwater (DUMAND, AMANDA, NESTOR) neutrino detectors or telescopes.

\section{Hot or cold}

Hot dark matter consists of particles which were relativistic when they separated (froze out) from matter in an early phase of the universe. Hot relics such as light neutrinos have number densities comparable to photons. Cold dark matter, on the other hand, is made of heavy particles which were already non-relativistic at freeze-out. The relic abundance of these particles depends inversely upon their annihilation cross-sections, and is significantly less than that of photons. Cold relics could be axions or WIMP's. Cosmological models predict that hot and cold dark matter scenarios have different effects on the development of the large-scale structures in the universe and on the formation of galaxies. For example, in a hot dark matter dominated universe, primordial density fluctuations occur only at very large scales (e.g., superclusters) because the particles are too hot to a permit clumping on smaller scales. These superclusters then fragmented into galaxies so galaxy forma-

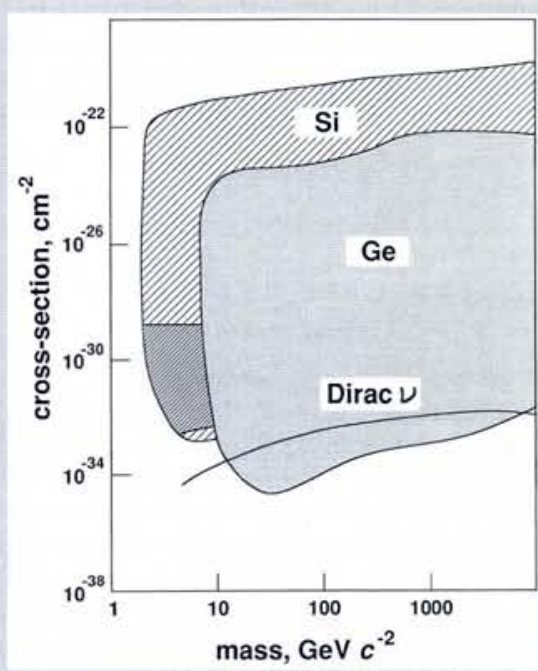

Exclusion zones for dark matter particles for Si and Ge detectors [Caldwell D.O. et al., Phys. Rev. Lett. 61 (1988) 510; Caldwell D.O., et al., Phys. Rev. Lett. 65 (1990) 1305]. A Caltech-Neuchâtel-Paul Scherrer Institute collaboration has obtained similar results [Reusser $R$. et al., Phys. Lett. B 255 (1991) 143]. The line labelled "Dirac v" indicates the values one would expect if the galactic halo consists of massive neutrinos. tion is a relatively recent phenomenon. In contrast, cold dark matter particles allow primordial clustering at much smaller scales (e.g., galaxies, dwarf galaxies) because they are non-relativistic at freeze-out. Galaxy formation then occurs rather early on, which is in better agreement with observations. Although the question of a hot or a cold dark matter dominated universe remains open, many cosmologists and particle physicists favour the latter because it solves two problems at the same time, namely dark matter and the grand unification of elementary particles. But the universe is perhaps a cocktail mixed from hot and cold dark matter

[1] Fich M. and Tremaine S., Ann. Rev. Astron. Astrophys. 29 (1991) 409.

[2] Milgrom M., Astrophysics J. 270 (1983) 365; Beckenstein J.D. and Milgrom M., Astrophysics J. 286 (1984) 7.

\section{Examples of Low-Threshold Cryogenic Detectors}

\section{Superheated Superconducting Granule (SSG) detector}

Millions of $10-20 \mu \mathrm{m}$ in diameter superconducting granules suspended in a dielectric granulate; detector surrounded by a single SQUID pick-up loop.

Based on WIMP-induced phase transitions (superconducting-normally conducting)

Prototype (50-100 g Sn granules) under construction (Bern Univ./LAPP, Annecy/Paul Scherrer Institute, Zurich) for testing at the Bern Underground Lab.

\section{Calorimeters with semiconducting thermometers}

Typically $20-100 \mathrm{~g}$ alumina crystal absorber at $20 \mathrm{mK}$ with a thermometer (usually neutron transmutation doped - NTD - Ge thermistor) attached.

Background tests started by a French group in the Frejus tunnel.

\section{Calorimeters with superconducting thermometers}

Crystal calorimeter with a superconducting thin film as the thermometer

Under development (Stanford Univ.; Max-Planck); dark matter detector under study.

Simultaneous phonon and ionization detector

A disc of ultrapure p-type Ge $(58 \mathrm{~g})$, with several NTD Ge thermistors attached (Univ. of California / Stanford Univ.).

Ionization caused by an event collected by means of a bias voltage between contacts on the faces of the disc.

Dark matter experiment in preparation. 